\title{
Insights into the Mechanism for Vertical Graphene Growth by Plasma-Enhanced Chemical Vapor Deposition
}

Sun, Jie; Rattanasawatesun, Tanupong; Tang, Penghao; Bi, Zhaoxia; Pandit, Santosh; Lam, Lisa; Wasen, Caroline; Erlandsson, Malin; Bokarewa, Maria; Dong, Jichen

Total number of authors:

13

Published in:

ACS Applied Materials and Interfaces

Link to article, DOI:

10.1021/acsami.1c21640

Publication date:

2022

Document Version

Publisher's PDF, also known as Version of record

Link back to DTU Orbit

Citation (APA):

Sun, J., Rattanasawatesun, T., Tang, P., Bi, Z., Pandit, S., Lam, L., Wasen, C., Erlandsson, M., Bokarewa, M., Dong, J., Ding, F., Xiong, F., \& Mijakovic, I. (2022). Insights into the Mechanism for Vertical Graphene Growth by Plasma-Enhanced Chemical Vapor Deposition. ACS Applied Materials and Interfaces, 14(5), 7152-7160. https://doi.org/10.1021/acsami.1c21640

\section{General rights}

Copyright and moral rights for the publications made accessible in the public portal are retained by the authors and/or other copyright owners and it is a condition of accessing publications that users recognise and abide by the legal requirements associated with these rights.

- Users may download and print one copy of any publication from the public portal for the purpose of private study or research.

- You may not further distribute the material or use it for any profit-making activity or commercial gain

- You may freely distribute the URL identifying the publication in the public portal 


\section{Insights into the Mechanism for Vertical Graphene Growth by Plasma-Enhanced Chemical Vapor Deposition}

Jie Sun,* Tanupong Rattanasawatesun, Penghao Tang, Zhaoxia Bi, Santosh Pandit, Lisa Lam, Caroline Wasén, Malin Erlandsson, Maria Bokarewa, Jichen Dong, Feng Ding,* Fangzhu Xiong, and Ivan Mijakovic

Cite This: ACS Appl. Mater. Interfaces 2022, 14, 7152-7160

Read Online

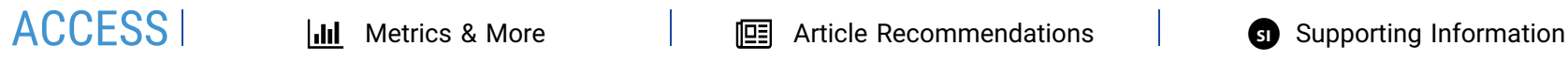

ABSTRACT: Vertically oriented graphene (VG) has attracted attention for years, but the growth mechanism is still not fully revealed. The electric field may play a role, but the direct evidence and exactly what role it plays remains unclear. Here, we conduct a systematic study and find that in plasma-enhanced chemical vapor deposition, the VG growth preferably occurs at spots where the local field is stronger, for example, at GaN nanowire tips. On almost round-shaped nanoparticles, instead of being perpendicular to the substrate, the VG grows along the field direction, that is, perpendicular to the particles' local surfaces. Even more convincingly, the sheath field is screened to different degrees, and a direct correlation between the field strength and the VG growth is observed. Numerical calculation suggests that during the growth, the field helps accumulate charges on graphene, which eventually changes the cohesive graphene layers into separate three-dimensional VG flakes. Furthermore, the field helps attract charged precursors to places sticking out from the substrate and makes them even sharper and turn into VG. Finally, we demonstrate that

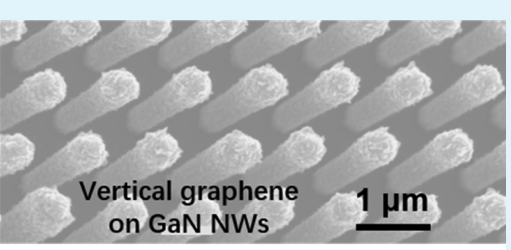
the VG-covered nanoparticles are benign to human blood leukocytes and could be considered for drug delivery. Our research may serve as a starting point for further vertical two-dimensional material growth mechanism studies.

KEYWORDS: vertical graphene, plasma-enhanced chemical vapor deposition, GaN nanowires, nanoparticles, 2D materials

\section{INTRODUCTION}

Vertically oriented graphene (VG) or so called carbon nanowalls are three-dimensional (3D), self-supported, interconnected networks of free-standing graphene sheets, whose orientations are approximately perpendicular to the substrate surface. Besides the fact that it possesses extraordinary properties of ordinary graphene, VG itself also has unique properties of high specific surface area, mechanical stability, open reactive graphene edges, easy functionalization, and special optical, thermal, and electrical properties. ${ }^{1-7}$ Due to these properties, VG has a high potential in a wide range of applications. It has caught a lot of interest in various sectors such as field emission, gas- and bio-sensors, blackbody coating, spintronics, and so forth. ${ }^{8-14}$

Although it has been reported that VG can be grown by radio frequency sputtering, ${ }^{15}$ plasma-enhanced chemical vapor deposition (PECVD) is by far the dominant technique to achieve the VG synthesis. ${ }^{11}$ PECVD provides several advantages compared to other techniques, including a lower substrate temperature, a higher growth rate, and a better control on nanostructure ordering due to the presence of energetic electrons, excited molecules/atoms, free radicals, photon, and other active species in the plasma region. Compared with thermal CVD which is based on neutral gas chemistry, PECVD is a more complex process, which can manipulate the morphology and structure of VG by altering the plasma source and adjusting a series of parameters. ${ }^{16-18}$

The growth mechanism of VG by PECVD has been studied and explained by many groups, but it still has some questions left to be answered. There are many factors whose roles have not been clearly revealed, such as the internal stress building up during the growth, the feature of anisotropic growth of VG, and the local electric field introduced by the plasma sheath in PECVD. ${ }^{1}$ Some authors think that the growth starts from the mismatch of the graphitic layer in the "carbon onion" and/or buffer layer, which serves as the nucleation center. ${ }^{11}$ Then, the VG continues its growth by surface diffusion after the deposition of carbonic atoms which are dissociated from the precursor gas in the plasma. Meanwhile, the surface of VG can also be etched with the atomic hydrogen in the growth

Received: November 8, 2021

Accepted: December 29, 2021

Published: January 10, 2022 


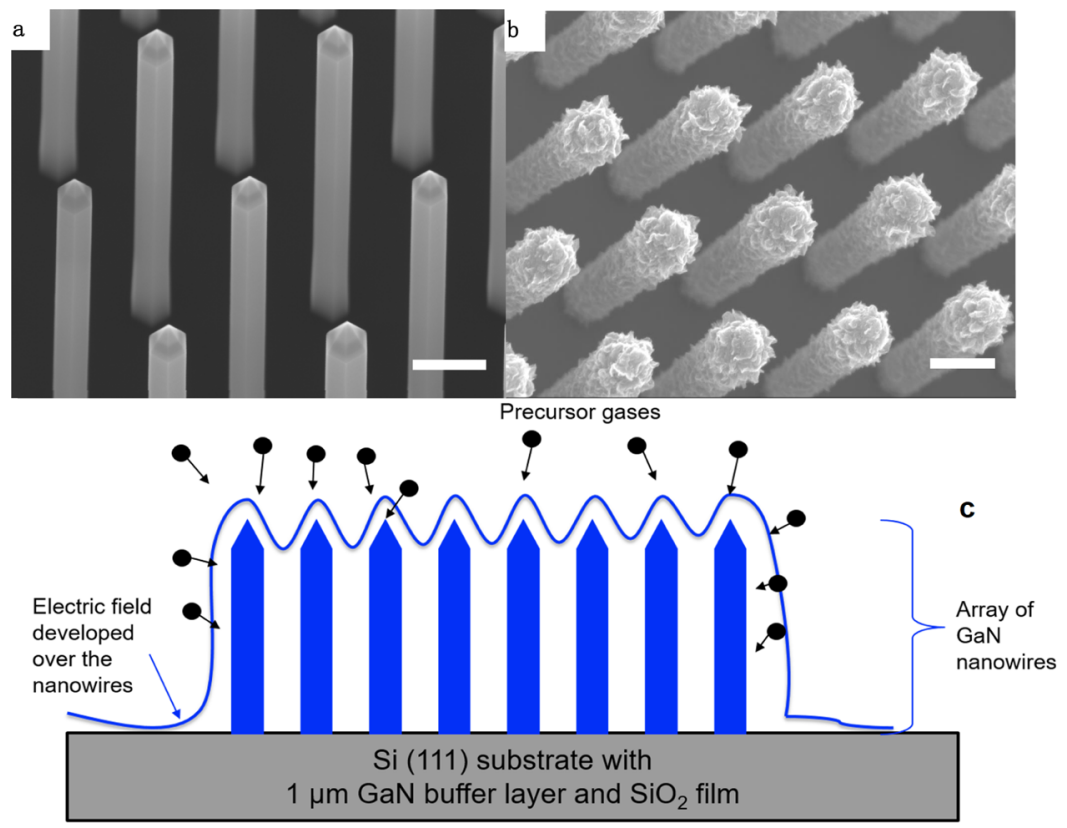

Figure 1. SEM images of GaN nanowires (a) before and (b) after the VG growth. In (b), it is seen that the VG coating on the GaN nanowires has a dominant effect of the local electric field, making the VG denser and longer in the tip region of the nanowires than on its body. Scale bar: 0.4 (a) and $0.5 \mu \mathrm{m}(\mathrm{b})$. The schematic illustration of the strong local electric field around the sharp tips and its enhancement effect on the VG growth is shown in (c).

chamber. ${ }^{19}$ Zhao et al. have developed a continuum model describing the mentioned mechanism and explaining the convergence tendency at the edges of the VG sheets. ${ }^{20}$ On the other hand, there are also evidences implicating that the electric field may be important in promoting the growth of VG. ${ }^{21}$ Controlling the coverage of the electric field can be used as a fabrication technique for patterning the VG in nanodevices. ${ }^{22}$ However, these studies are generally not conclusive. For example, in ref 22, it is observed that VG prefers to grow on $\mathrm{Au}$ rather than on $\mathrm{SiO}_{2}$ surfaces, and it is explained by the presence of much stronger electric field in the vicinity of $\mathrm{Au}$ as compared with $\mathrm{SiO}_{2}$. Nevertheless, it could also be possibly explained by a much better catalysis effect on the gold surface compared with $\mathrm{SiO}_{2}$. Therefore, up till now, to our knowledge, there is no convincing and direct evidence showing that electric field does promote the growth of VG and explaining its mechanism in detail. We do believe that the central role in the growth mechanism of VG is played by the electric field. We think that the carbonic ions diffusing along the surface of VG are pulled to the direction of the relatively stronger electric field in the structure, thus resulting in the denser and longer VG growth as compared to the other areas where the fields are weak.

In this work, we will demonstrate more and much stronger supporting evidences confirming the effect of local electric field in enhancing the growth of VG on flat substrates and on nanostructures. VG has been first deposited by PECVD on GaN sharp-tipped nanowires to reveal the relation between the effect of local electric field and the VG growth. We have proposed a model to explain the strong-electric field-enhanced VG growth on sharp-tipped GaN nanowires from a cold-walled PECVD chamber. Another experiment is set up to observe the screening effect of local electric field using stainless-steel meshes that can partly block the electric field formed in the plasma sheath while operating the PECVD. The field on the substrate surface is screened to different extents, and the growth results which are studied by using scanning electron microscopy (SEM) are compared. The positive effect of the field on VG growth is again confirmed without ambiguity. We have also succeeded in fabricating a novel material, which is VG-coated silica nanoparticles. Previously, there was an attempt to wrap nanoparticles with graphene oxide ${ }^{23}$ for drug delivery application. However, to our knowledge, there has been no report on wrapping nanoparticles with VG. It is found that the $\mathrm{VG}$ grows perpendicular to the $\mathrm{SiO}_{2}$ nanoparticle surfaces instead of growing perpendicular to the substrate, which indicates that the VG growth direction is in line with the local field. We have experimentally found that the VG growth actually starts from flat graphene growth. By virtue of the electric field of the plasma, charges accumulate on the graphene flakes. Numerical calculation is carried out, and it is found that due to the Coulombic repulsive force, the flakes change their growth direction and stick out vertically. Also, because sharper features on the substrate are places where the field is stronger, they attract more active species that promote the growth, which makes the features even sharper. This positive feedback loop further boosts the VG growth. Thus, the theoretical results explain the experimental findings well. Finally, for applications, we have cultured human peripheral blood mononuclear cells (PBMCs) on top of our substrates decorated with the VG-coated nanoparticles on their surfaces. The biological interaction between the cells and the VG-coated particles is observed. Despite the observed interaction with VG, we found no harmful consequences to the cells with respect to viability and cytokine production, which indicates its potential application in drug delivery. Our results can be seen as a systematic investigation of the electric field influence on the VG growth with a specific value to material growth scientists and engineers working in the field and can be extended to the vertical growth of two-dimensional (2D) materials beyond graphene. 

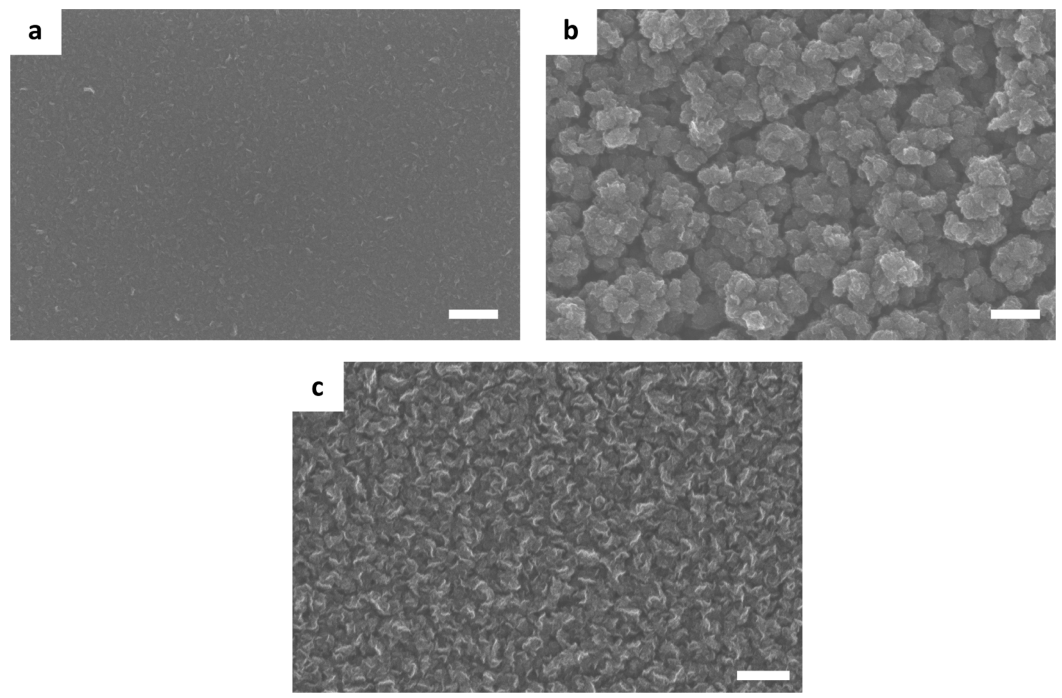

Figure 2. SEM images of the VG grown on the $2 \mathrm{in} . \mathrm{SiO}_{2} / \mathrm{Si}$ substrate surface when the electric field is screened using stainless-steel meshes to different degrees. (a) Typical VG growth in region I in Figure S3, where the electric field is almost totally screened using a small mesh with the hole size of about $140 \mu \mathrm{m}$. An almost entire inhibition of VG growth is observed. (b) Electric field is partly blocked using a metal mesh with the hole size of about $400 \mu \mathrm{m}$, corresponding to region II in Figure S3. There is 3D growth, but with no sharp edges and also the "VG" is grown as clusters rather than "free-standing" sheets. (c) In region III where there is no screening, the VG growth is back to normal and flower-like vertical flakes are observed as expected. Scale Bar: $0.5 \mu \mathrm{m}$ for all three images.

\section{RESULTS AND DISCUSSION}

2.1. VG Growth on GaN Nanowires. In corona discharge theory, charges tend to accumulate toward sharp edges, and electric field is the strongest where the radius of curvature is small. Therefore, trying to grow VG on sharp surfaces is the best way to clarify whether the local electric field is indeed a factor for promoting the VG growth or not. Thus, in order to study the effect of electric field and fully understand its growth mechanism, we grow arrays of $\mathrm{GaN}$ nanowires on $\mathrm{GaN} / \mathrm{Si}$ substrates from nanoscale openings patterned in a $\mathrm{SiN}_{x}$ mask by electron beam lithography, as shown in Figure 1a. The nanowires grow selectively from the $\mathrm{SiN}_{x}$-free openings, and the nanowires have a height of about $1.5 \mu \mathrm{m}$ and a width of about $200 \mathrm{~nm}$. After standard organic cleaning, this nanowire pattern is used for the VG growth. The VG is then deposited in our cold-walled PECVD chamber. A typical Raman spectrum is shown in Figure S1, which is taken from a VG sample grown for $10 \mathrm{~min}$ with our standard parameters as reported elsewhere. ${ }^{24}$ Based on the G, 2D, and D Raman peaks, it can be concluded that the grown VG is $\mathrm{sp}^{2}$-hybridized. Figure $1 \mathrm{~b}$ shows SEM images after the growth of VG. It can be seen that the VG grows all over the wires. From Figure S2, it is clear that the $\mathrm{VG}$ also grows on the $\mathrm{SiN}_{x}$ mask between the nanowires on the substrate. However, the VG flakes at the tips of the nanowires are much denser and longer than those grown on the nanowire bodies (see Figure 1b). The VG flakes at the tips are measured to be about 100-150 nm in length. In Figure S2, the flow rate of the $\mathrm{C}_{2} \mathrm{H}_{2}$ precursor gas and the time used in the growth is modulated. It shows that when the flow rate and growth time are high enough, the effect of electric field on the VG growth can be observed distinctly.

The observed phenomenon can be well explained by the enhanced electric field around the tips. When the nanowire structure is covered with the plasma sheath in the PECVD machine, it will develop a local electric field profile based on its geometry. For our highly crystalline GaN nanowires, because of their hexagonal pyramid-shaped apexes as shown in Figure 1a, they naturally hold the strongest electric field at their sharp tips, which can result in attracting carbon ions in the plasma for adsorption and diffusion there, leading to an enhanced VG growth mode. It was, thus, expected to have the densest and also the longest $\mathrm{VG}$ growth at these pointing tips. We note that because this type of semiconductor nanowires is highly crystalline and forms highly ordered arrays, it is probably the best candidate to study the electric field effect. To the best of our knowledge, this is the first experiment of growing VG on GaN semiconductor nanowires. VG flakes have been grown on carbon nanotubes as well. ${ }^{25}$ However, because today's technology for producing carbon nanotubes is not as neat as that for semiconductor nanowires and, most importantly, carbon nanotubes generally have round ends, ${ }^{26}$ the results of the electric field effect on the VG growth there are not very conclusive.

2.2. Electric Field Screening Effect with StainlessSteel Meshes. In our experiment, we have found that if remote plasma instead of local plasma is used in the PECVD, the VG can be hardly grown. This is an indication that in order to initiate the VG growth, the samples have to be immersed in the plasma sheath, where the electric field is the strongest. In the following, we design another experiment to investigate deeper on the effect of electric field influencing the growth. In the setup, two stainless-steel meshes with different hole sizes are used to screen part of the electric field. In this experiment, the plasma is local where its electrode is located directly below the graphitic heater plate. The substrate, which is a 2 in. silicon wafer with $400 \mathrm{~nm} \mathrm{SiO}{ }_{2}$ coating, is placed on the heater. The plasma electrode is powered with positive voltage while the heater is grounded. The huge voltage difference is then used to ignite the plasma, and the electric field from the plasma sheath is formed from slightly above the heater. The two stainlesssteel meshes are placed less than $1 \mathrm{~mm}$ above the heater and cover some part of the substrate, as shown in Figure S3. The sample area can be divided into three regions (see Figure S3c). Region I is covered with mesh 1 with small holes $(140 \mu \mathrm{m}$ diameter), region II is covered with mesh 2 with slightly bigger 
holes (400 $\mu \mathrm{m}$ diameter), and region III is the uncovered area. Due to the discharge effect, where the sparkles are vividly seen in Figure S3b, some areas have not been effectively screened, which means that at some spots, electric fields are leaked to the sample beneath the meshes. Notwithstanding this, there do exist some areas that have been successfully screened out of any electric field, as outlined by the dashed lines in Figure S3c. The SEM images of the VG after the PECVD growth are summarized in Figure 2, where Figure 2a is taken from region I. It is the area where most of the electric field is screened out using a metal mesh with small holes. The growth of VG is almost totally inhibited by the absence of the electric field influence. Because the mesh is only screening out the electric field, the precursor gas can still reach the substrate surface, which leads to the growth of ordinary few-layered flat graphene. Although for the area in Figure $2 \mathrm{~b}$, the electric field is also blocked but with somewhat more leakage due to the bigger size of the mesh holes, the image shows that there is some $3 \mathrm{D}$ growth of VG-like structures. However, they are missing the feature of sharp edges, and they also grow as clusters instead of "free-standing" vertical sheets. Both growth results demonstrate the importance of local electric field that is heavily influencing the growth of VG. On the $\mathrm{SiO}_{2} / \mathrm{Si}$ substrate where it is not covered with any steel mesh, the typical SEM image is represented in Figure 2c. Not surprisingly, the VG in this area shows a network of standing graphene flakes with the feature of sharp edges, which is expected for a standard VG growth.

2.3. VG Growth on Nanoparticles. Another experiment we will carry out is to grow VG on nanoparticles. To our knowledge, this has not been carried out before. One of the motivations for this experiment is to further study the local electric field effect. When the VG is grown on the nanoparticles, it could take two possible orientations as schematically drawn in Figure 3a. On the left part of this figure, the VGs are pointing upward, which at first glance seems to be logical. However, because the electric field in the plasma follows the shape of objects that are immersed in the sheath, the local field direction should be perpendicular to the surfaces of the particles. Hence, the profile on the right part of Figure $3 \mathrm{a}$ is expected. Described below is our actual verification of this hypothesis.

$\mathrm{SiO}_{2}$ nanoparticles dispersed in isopropanol are drop-coated on the $\mathrm{SiO}_{2} / \mathrm{Si}$ substrate and left for drying overnight. These commercial nanoparticles have an approximately round or hexagonal shape with a typical size of $0.45-0.5 \mu \mathrm{m}$ and are self-organized on the substrate as shown in Figure $3 \mathrm{~b}$. We then grow VG on the sample by the same PECVD method. With the flow rate and growth time being carefully tuned, the VG height can be well controlled. Figure $3 \mathrm{c}-\mathrm{f}$ shows the SEM images of the VG-coated $\mathrm{SiO}_{2}$ nanoparticles with different VG heights. The growth parameters are written in the figure caption. As expected, higher flow rates and longer deposition time lead to longer VG flakes. Importantly, it also shows that the growth of the VG on the nanoparticles (see Figure 3e,f) is in the normal direction with respect to the surfaces of the particles or, in other words, in the same direction of the electric field formed on their surfaces. We have also grown VG on $\mathrm{Fe}_{3} \mathrm{O}_{4}$ nanoparticles $(\sim 10 \mathrm{~nm}$ in size $)$ in the same manner, and similar results are observed.

2.4. Deeper Look into the Electric Field-Assisted Growth Mechanism of VG. Based on the experimental results we have got, we are able to dig deeper into the growth a
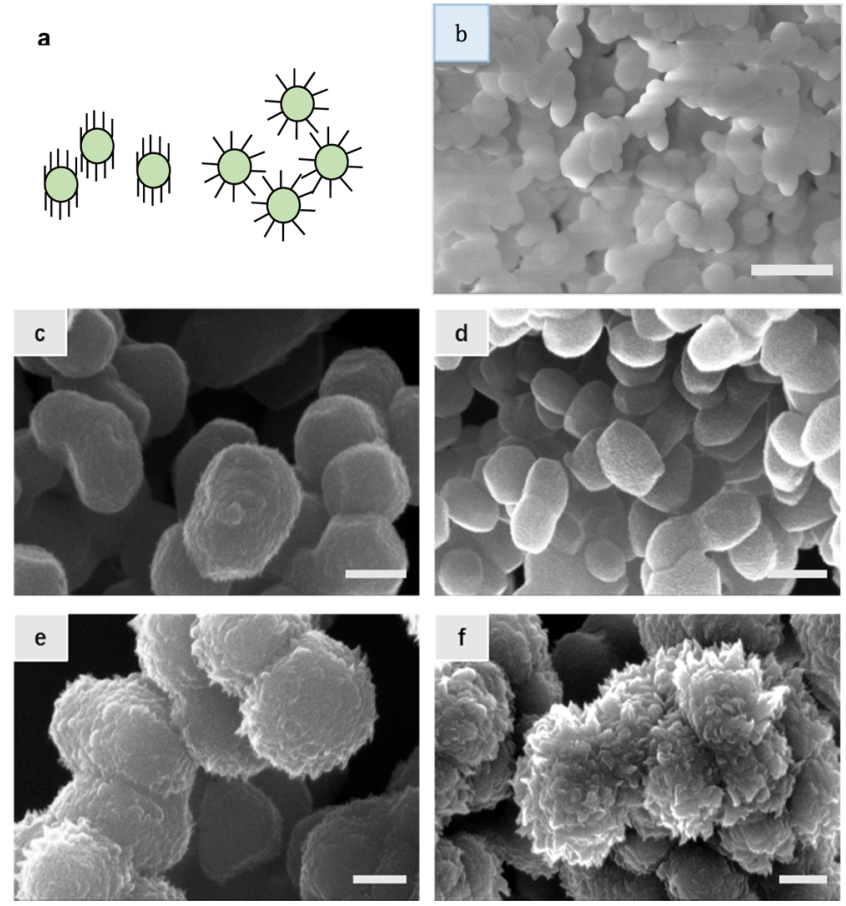

Figure 3. (a) Schematic illustration of two possible growth modes of the VG on nanoparticles. On the left, the flakes are perpendicular to the substrate. On the right, the flakes are alongside the normal directions of the nanoparticles, that is, the directions of the local field. The latter growth mode is confirmed by the experiments. (b) SEM image of the $\mathrm{SiO}_{2}$ nanoparticles that are drop-coated on the substrate surface. The particles are about $450-500 \mathrm{~nm}$ in size. (c-f) SEM images of the VG grown on the silica nanoparticles with different flow rates of $\mathrm{C}_{2} \mathrm{H}_{2}$ and growth times. The flow rates and growth times are: (c) $5 \mathrm{sccm}$ and $3 \mathrm{~min}$, (d) $5 \mathrm{sccm}$ and $5 \mathrm{~min}$, (e) $15 \mathrm{sccm}$ and $3 \mathrm{~min}$, and (f) $15 \mathrm{sccm}$ and $5 \mathrm{~min}$. Scale bar: (b) $2 \mu \mathrm{m}$, (c) $500 \mathrm{~nm}$, (d) 1 $\mu \mathrm{m}$, (e) $400 \mathrm{~nm}$, and (f) $500 \mathrm{~nm}$.

mechanism of VG. It is known that when the VG grows, it usually starts with horizontal graphene growth, ${ }^{27}$ which can be regarded as a buffer layer. This is a logical and natural scenario. However, there has not been very clear and direct observation of such a horizontal layer in the literature. Here, we present a concrete evidence of the horizontal graphene underneath the VG during the initial growth stage. Figure 4 shows the SEM image of a VG film grown on a $300 \mathrm{~nm} \mathrm{SiO} 2 / \mathrm{Si}$ flat substrate using our standard recipe. Figure $4 \mathrm{~b}$ shows the SEM image (top view) of the VG, where the graphene flakes are tightly connected to each other, most likely covalently bonded, forming a porous film. Therefore, the VG film is mechanically rather strong. It is known that in a liquid environment, the surface tension pulls toward any solid that the liquid is in contact with. For our VG, however, we have found that even if it is directly taken out of water and blow-dried with $\mathrm{N}_{2}$, the graphene will not collapse. It even keeps its integrity when it is mechanically delaminated from the substrate (see Figure $4 \mathrm{~b}$, where both the top and back sides are visible). When the focus is set to the topside, the typical VG morphology is observed, as shown in the enlarged micrograph of Figure 4a. When focusing on the backside (Figure 4c), it is seen to be super flat, meaning that all the vertical flakes are rooted in a horizontal graphene film. Observing the cross-section, the film thickness is on the order of few hundred nm. Figure 4 points out an important fact 


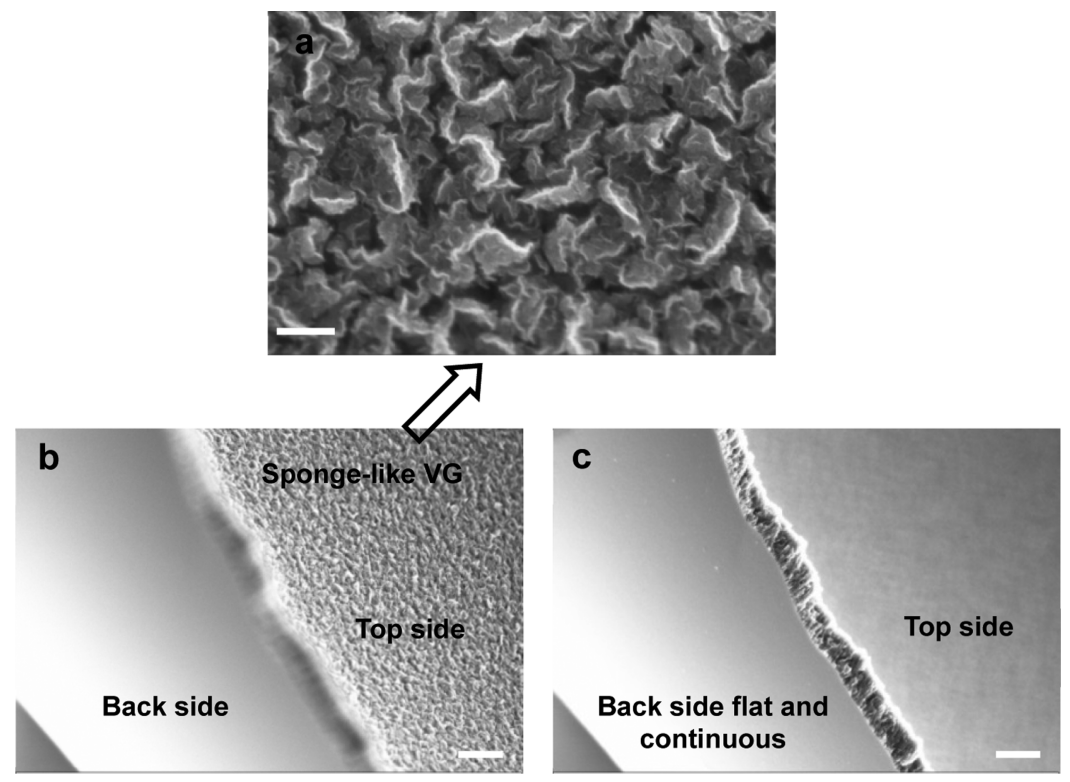

Figure 4. (a) SEM micrograph of a VG film grown by PECVD on the $\mathrm{SiO}_{2} / \mathrm{Si}$ substrate. The flakes are rooted in the substrate and stick out vertically in 3D space. $(b, c)$ SEM images with focus on the top (b) and back (c) side. They show that the porous VG film is mechanically rather strong and can be peeled off from the substrate. On the top side, numerous covalently bonded graphene flakes are seen, whereas the backside is totally flat and continuous. Most importantly, it directly proves that this type of graphene first grows horizontally, and when the thickness slightly increases, the growth mode changes to the vertical growth. The SEM micrographs in (b,c) are taken from an angle. Scale bar: $200 \mathrm{~nm}$ in (a) and 1 $\mu \mathrm{m}$ in $(\mathrm{b}, \mathrm{c})$

that the deposition indeed starts from a horizontal growth mode.

In plasma, electrons moves much faster than positively charged ions. Therefore, what usually happens is that electrons will transfer to the substrate and charge it negatively. An electric field is thus produced at the interface between the substrate and the plasma at equilibrium. This layer is called the Debye sheath, and the thickness of such a layer is called Debye length. Because the VG growth starts with horizontal graphene deposition, in plasma, these graphene layers are negatively charged in the sheath, as schematically shown in Figure 5a. We propose that the most important reason for the growth mode to change from $2 \mathrm{D}$ to $3 \mathrm{D}$ is the charge accumulation. By virtue of the Coulombic repulsive force, the graphene layers are split and start to point upward. We have carried out a simulation on this growth mechanism. Figure $5 b$ shows the split distance between the graphene layers as a function of the charge. Eight charges are enough to separate the two neighboring graphene layers by $\sim 3 \mathrm{~nm}$ (Figure 5b,c). Clearly, the morphology of the originally horizontal multilayer graphene is heavily influenced by its charge status. Figure $5 \mathrm{~d}$ shows the Mulliken charge distribution on the graphene flakes. It is found that with the increase in the system charging value, the added electrons tend to distribute around the circumference.

We also propose another important VG growth mechanism that is based on the local electric field as well. When the $2 \mathrm{D}$ to $3 \mathrm{D}$ growth transition happens as described above, some places start to appear slightly higher than others. Therefore, the local field is stronger at these higher points because of the smaller radius of curvature. The decomposed precursor consists of charged ions, which are hence directed toward these positions by the electric field. As a result, the VG grows more in these areas as compared to other places. Little by little, they become even sharper and protruded, which in turn makes the field even stronger, forming a positive feedback loop. The two growth mechanisms are both electric field-initiated, and they work simultaneously in the growth chamber, facilitating the VG deposition. This explains why when the electric field is screened out, the VG growth literally ceases. Also, if remote PECVD is used instead as the samples are not immersed in the plasma sheath where the electric field is strong, although the precursors can still be effectively cracked, the VG growth is not feasible any more.

2.5. Interaction of VG-Covered Nanoparticles with Human Cells for Potential Drug Delivery Applications. It is proposed that graphene has a high potential in drug delivery. $^{23,28}$ However, up till now, there are still important issues remaining unclarified. For example, whether graphene is harmful to human cells remains arguable. Nanoparticles are a type of traditional materials used in drug delivery. VG flakes have a high surface-to-volume ratio and can be grown virtually on any stable material that withstands high temperature. Therefore, we propose to combine nanoparticles with VG for drug delivery applications. Due to the local electric field-based growth mechanism, the VG flakes stick out toward every direction (see Figure 3e,f), which is in favor of drug delivery. The high specific area of $\mathrm{VG}$ may increase $\mathrm{SiO}_{2}$ particles' functionality. In the following, we demonstrate that they are indeed human cell-friendly.

We first coat $\mathrm{SiO}_{2}$ nanoparticles with height-controllable VG. PBMCs are cultured on the $\mathrm{SiO}_{2} / \mathrm{Si}$ substrate that is deposited with VG-coated $\mathrm{SiO}_{2}$ nanoparticles. We find that the $\mathrm{VG}$, even with its sharp edges, does not endanger the PBMCs. Figure 6 shows the interaction between the cells and the VGcoated $\mathrm{SiO}_{2}$ particles. The cell morphology is clearly visible and preserved. The cells interact and engulf the particles presenting normal physiological function. In Figure $6 a$, the cell covers or internalizes a cluster of $\mathrm{SiO}_{2}$ nanoparticles. In Figure $6 \mathrm{~b}$, the cell forms sprouts, reaches out, grasps, and internalizes a large number of nanoparticles. Interestingly, the cell 

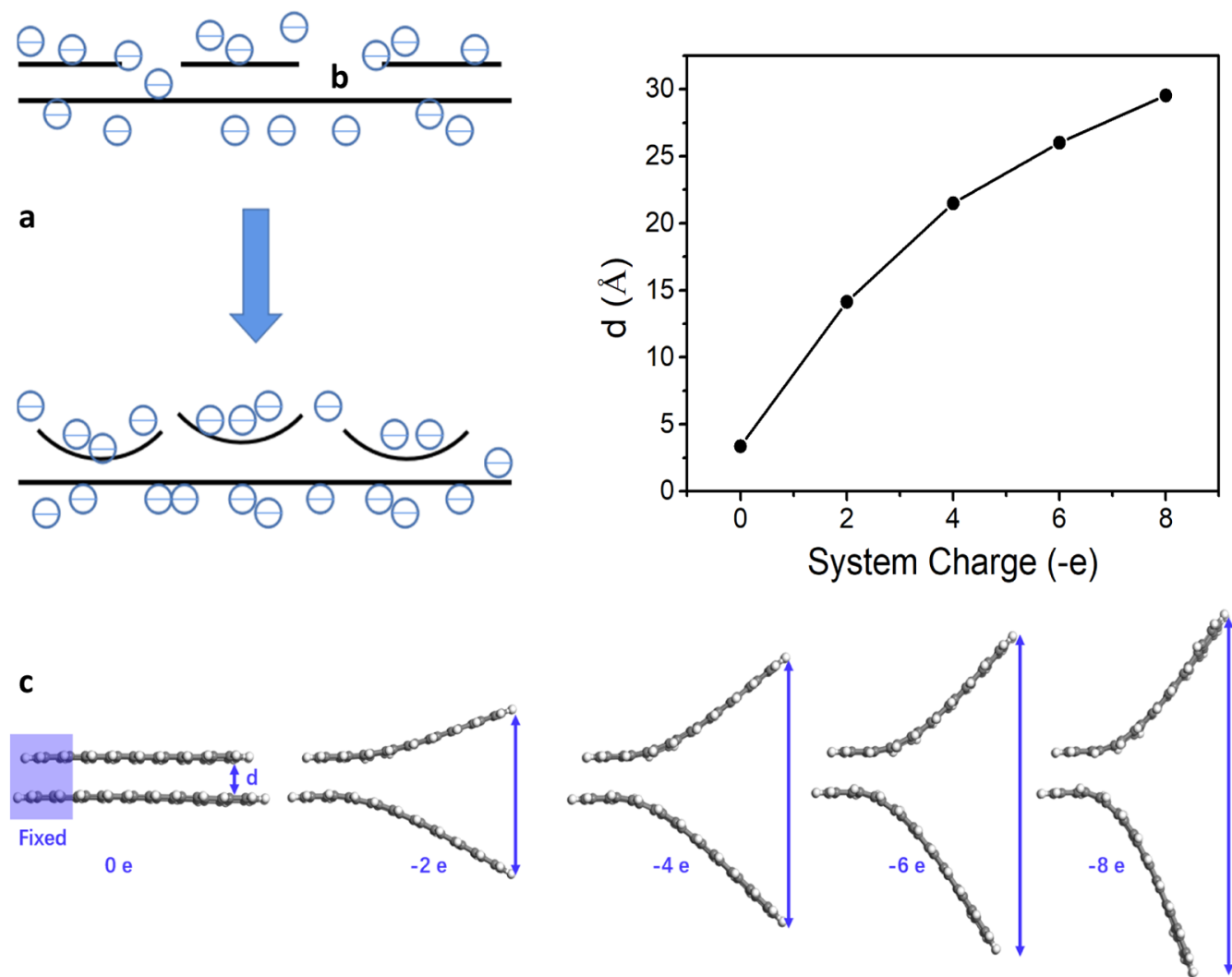

d

\section{:

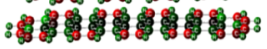

Mulliken charge distribution

$0 \mathrm{e}$

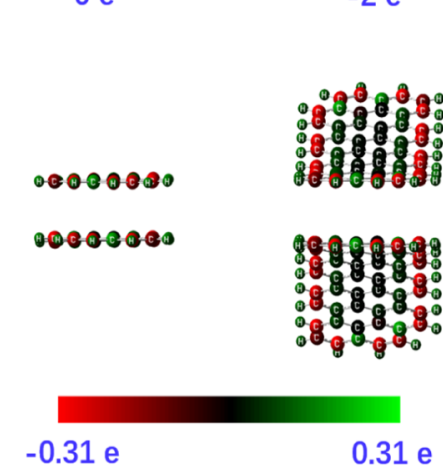

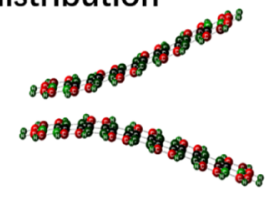

$-2 \mathrm{e}$

$0.31 \mathrm{e}$

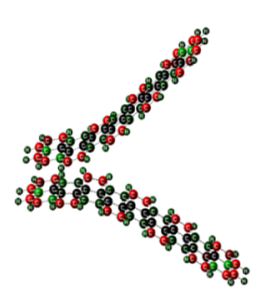

-4 e
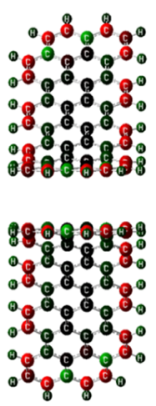

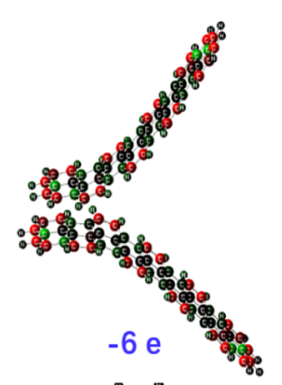

0

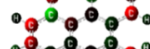

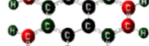

.9 .0 .0

$18^{\circ}$

ติ989.

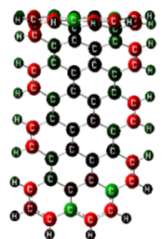

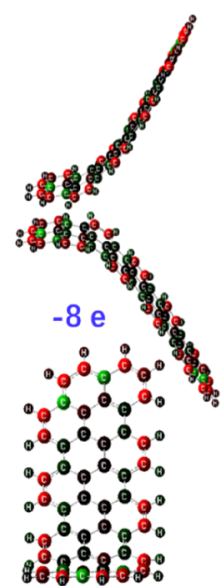

-gazagas

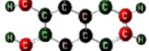
$\therefore \circ: 00$ 0

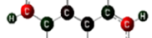
$00_{0} \theta_{0} \theta^{\circ}$

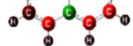

Figure 5. (a) Schematic demonstration of the change from 2D to 3D growth due to the charge accumulation. When the charge density is high enough, the Coulombic force would overcome the van der Waals interaction in multilayer graphene, leading to the formation of VG. (b-d) Simulation results. (b) Distance between the two graphene tips plotted as a function of the system charging value. (c) Same data, showing that with the increase in the system charging value, the distance between the tips increases significantly due to the electrostatic repulsive force between the tips. (d) Simulated Mulliken charge distribution. With the increase in the system charging value, the Mulliken charges (added electrons) tend to distribute near the circumference.

demonstrates certain ability to change its position even after "eating" the nanoparticles. In Figure $6 b$, we can see a dark border around the cell. It is because the particles therein have been removed by the cell, and the VG-free surface of the $\mathrm{SiO}_{2}$ /
Si substrate is exposed. This procedure is schematically illustrated in Figure 6c,d. This experiment demonstrates that the PMBCs are still healthy after interacting with the VGcoated $\mathrm{SiO}_{2}$ particles. This result expands the potential 

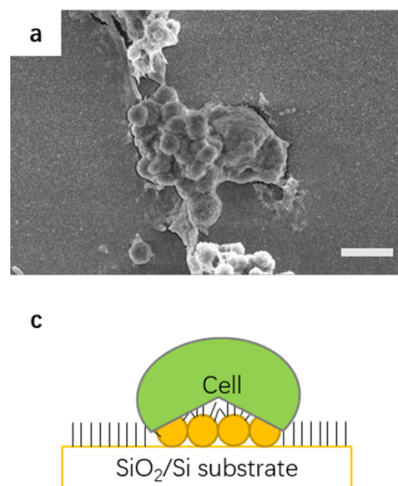

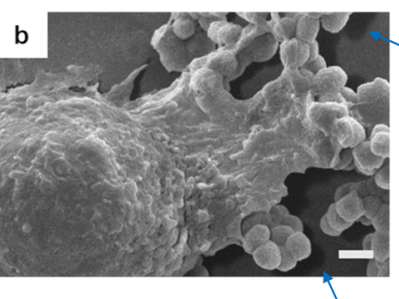

d

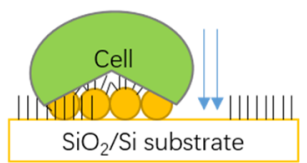

Figure 6. (a) SEM image showing that a cell interacts with a cluster of VG-covered nanoparticles either by covering or internalizing the nanoparticles. The shape of the nanoparticle cluster may be seen under the cell membrane. (b) Cell is interacting with a cluster of nanoparticles. The cell forms sprouts which are reaching out for the nanoparticle clusters. (c) Schematic illustration of the PBMC covering or internalizing nanoparticles on the $\mathrm{SiO}_{2}$ substrate. (d) Schematic illustration of the PBMC movement after the internalizing of nanoparticles. The arrows point at the area where the nanoparticles have been moved by the cell, which leaves dark borders in the SEM image in (b). Scale bar: 3 (a) and $1 \mu \mathrm{m}$ (b).

application of VG in the pharmaceutical field. Some additional SEM images can be found in Figure S4.

\section{CONCLUSIONS}

In summary, the mechanism of VG growth in PECVD has been systematically studied. The VG is deposited on GaN nanowires, and it is found that the VG prefers to grow at the sharp tips, where the local electric field is the strongest. Furthermore, the field is particularly screened by using metal meshes with different sizes of holes to verify the vital effect of the field. $\mathrm{On} \mathrm{SiO}_{2}$ nanoparticles, it is found that the VG always grows perpendicular to the surface of the particles, namely, along the direction of electric field. Theoretical calculation points out that when the VG grows, the electric field can accumulate electrons on the flakes, which eventually turns the growth mode from $2 \mathrm{D}$ into $3 \mathrm{D}$ by virtue of the Coulombic repulsion. Also, sharper points are places where the electric field is stronger, which will direct the charged precursor species to these points and promote the growth. Finally, we demonstrate a potential application for drug delivery using the VG-covered $\mathrm{SiO}_{2}$ particles, whose surface to volume ratio has been maximized. Previously, the understanding of VG growth mechanism had been very vague. Although the electric field is believed to play a role in the VG growth, the direct evidence of this role and exactly how it works has been missing in the literature. Our research unambiguously reveals the electric field-based growth mechanism and can be of a reference value to VG growth designers in the field. For example, although the precursors may be effectively cracked, remote PECVD may not work for the VG growth because the samples are placed outside of the intensive electric field area. Finally, we point out that our results may be useful as a guideline for exploring a family of vertically aligned $2 \mathrm{D}$ materials other than graphene. A typical example is h-BN, which is a $2 \mathrm{D}$ insulator. The growth mechanism is still under investigation, ${ }^{30}$ but vertical h-BN flakes have been realized by PECVD already. ${ }^{31}$ Vertical 2D semiconductors, for example,
$\mathrm{MoS}_{2}$ is also synthesized, although with methods other than PECVD. ${ }^{32,33}$

\section{EXPERIMENTAL SECTION}

4.1. PECVD Growth of VG. VG was grown on the substrate in a cold-walled low-pressure PECVD reactor (Black Magic, Aixtron). The substrate was heated to $775{ }^{\circ} \mathrm{C}$ and annealed for $1 \mathrm{~min}$ with the mixing of $20 \mathrm{sccm} \mathrm{H} \mathrm{H}_{2}$ gas and $1000 \mathrm{sccm} \mathrm{Ar}$ gas. The plasma is then turned on under a DC bias with the power of $75 \mathrm{~W}$. The plasma voltage is $800 \mathrm{~V}$ with the current limit of $0.5 \mathrm{~A}$. The actual growth was initiated by introducing $\mathrm{C}_{2} \mathrm{H}_{2}$ (usually $15 \mathrm{sccm}$ ) and maintained typically for $10 \mathrm{~min}$. After the growth, the system was evacuated to $<0.2$ mbar and cooled down.

4.2. Preparation of $\mathrm{GaN}$ Nanowires and $\mathrm{SiO}_{2}$ Nanoparticles. Pure GaN nanowires with no indium or aluminum compositions were grown by metal organic CVD on (0001)-oriented GaN/Si substrates [the $\mathrm{Si}$ is (111)-oriented] with a $30 \mathrm{~nm}$-thick $\mathrm{SiN}_{x}$ mask. Electron beam lithography was used to fabricate openings in the $\mathrm{SiN}_{x}$ mask, where the $\mathrm{GaN}$ nanowires grew.

The $\mathrm{SiO}_{2}$ nanoparticles were actually $\mathrm{Zr}$-SBA-15, a kind of mesoporous $\mathrm{SiO}_{2}$ nanoparticles often used in drug delivery. The powders were uniformly dispersed in isopropanol or acetone with ultrasonic treatment. Two or three drops of the solution were then drop-coated on the $1 \times 1 \mathrm{~cm}^{2} \mathrm{SiO}_{2} / \mathrm{Si}$ substrate and left overnight for drying.

4.3. Cell Culture. Human PBMCs were isolated from venous peripheral blood of healthy volunteers using density gradient separation on Lymphoprep (Axis-Shield PoC As, Norway). Cells were washed and resuspended in Iscove's medium (containing $1 \% \mathrm{~L}-$ glutamine, $5 \times 10^{-5} \mathrm{M} \beta$-mercaptoethanol, $50 \mu \mathrm{g} / \mathrm{mL}$ of gentamycin sulfate, and $10 \%$ fetal calf serum) at $2 \times 10^{6} / \mathrm{mL}$ and set on the slides coated with VG-coated $\mathrm{SiO}_{2}$ nanoparticles. Cell cultures were stimulated with LPS $(50 \mathrm{ng} / \mathrm{mL})$ for 24 and $72 \mathrm{~h}$ in a humidified atmosphere containing $5 \% \mathrm{CO}_{2}$ at $37{ }^{\circ} \mathrm{C}$. The PBMCs were fixated on the substrates. The samples were then coated with a $5 \mathrm{~nm}$ gold layer by sputtering before the SEM observation.

\section{THEORETICAL CALCULATION DETAILS}

All calculations were carried out by using the Gaussian09 package with the B3LYP hybrid functional method and 6$31 \mathrm{G}(\mathrm{d})$ basis. $^{29}$ As shown in Figures 5 and S5, two parallelly stacked graphene nanoribbon flakes were constructed to represent few-layered graphene in experiments. Different amounts of charges were added to the calculation model to investigate the charge effect on the structure of few-layered graphene. The structure was optimized with its one end fixed and the forces of all the other atoms relaxed to less than $2 \times$ $10^{-4}$ Hartree/Bohr.

\section{ASSOCIATED CONTENT}

\section{S1 Supporting Information}

The Supporting Information is available free of charge at https://pubs.acs.org/doi/10.1021/acsami.1c21640.

Typical Raman spectrum of the VG film confirming the graphitic structure, additional SEM images of the VG gron on $\mathrm{GaN}$ nanowires with different flows and times, photographs of the experimental setup for screening the electric field using stainless-steel meshes, additional SEM images showing the intimate interaction of the cells and the VG-coated particles, and atomic structures of the theoretical calculation model (PDF) 


\section{AUTHOR INFORMATION}

\section{Corresponding Authors}

Jie Sun - National and Local United Engineering Laboratory of Flat Panel Display Technology, College of Physics and Information Engineering, Fuzhou University, and Fujian Science \& Technology Innovation Laboratory for Optoelectronic Information of China, Fuzhou 350116, China; Department of Microtechnology and Nanoscience, Chalmers University of Technology, Göteborg 41296, Sweden; ๑ orcid.org/0000-0002-6479-7771; Email: jie.sun@fzu.edu.cn

Feng Ding - Centre for Multidimensional Carbon Materials, Institute for Basic Science, Ulsan National Institute of Science and Technology, Ulsan 44919, Korea; $\odot$ orcid.org/00000001-9153-9279; Email: f.ding@unist.ac.kr

\section{Authors}

Tanupong Rattanasawatesun - Department of Microtechnology and Nanoscience, Chalmers University of Technology, Göteborg 41296, Sweden

Penghao Tang - Key Laboratory of Optoelectronics Technology, College of Microelectronics, Beijing University of Technology, Beijing 100124, China

Zhaoxia Bi - Division of Solid State Physics and NanoLund, Department of Physics, Lund University, S-22100 Lund, Sweden; ๑ orcid.org/0000-0002-7024-7266

Santosh Pandit - Department of Biology and Biological Engineering, Chalmers University of Technology, Göteborg 41296, Sweden; ๑o orcid.org/0000-0002-8357-758X

Lisa Lam - Department of Rheumatology and Inflammation Research, University of Gothenburg, Göteborg 41346, Sweden

Caroline Wasén - Department of Rheumatology and Inflammation Research, University of Gothenburg, Göteborg 41346, Sweden

Malin Erlandsson - Department of Rheumatology and Inflammation Research, University of Gothenburg, Göteborg 41346, Sweden

Maria Bokarewa - Department of Rheumatology and Inflammation Research, University of Gothenburg, Göteborg 41346, Sweden

Jichen Dong - Centre for Multidimensional Carbon Materials, Institute for Basic Science, Ulsan National Institute of Science and Technology, Ulsan 44919, Korea

Fangzhu Xiong - Key Laboratory of Optoelectronics Technology, College of Microelectronics, Beijing University of Technology, Beijing 100124, China

Ivan Mijakovic - Department of Biology and Biological Engineering, Chalmers University of Technology, Göteborg 41296, Sweden; The Novo Nordisk Foundation Center for Biosustainability, Technical University of Denmark, 2800

Kgs. Lyngby, Denmark; @ orcid.org/0000-0002-8860-6853

Complete contact information is available at:

https://pubs.acs.org/10.1021/acsami.1c21640

\section{Notes}

The authors declare no competing financial interest.

No data sets were generated or analyzed in the current study.

\section{ACKNOWLEDGMENTS}

We thank the support from Fujian provincial projects (nos. 2021HZ0114, 2021J01583, and 2021L3004), Mindu projects (nos. 2021ZZ122 and 2020ZZ110), and the National Natural
Science Foundation of China (no. 62175032). We acknowledge support from the Novo Nordisk Foundation (NNF10CC1016517), the Independent Research Fund Denmark-FTP, and Nord Forsk to I.M.

\section{REFERENCES}

(1) Bo, Z.; Mao, S.; Han, Z. J.; Cen, K.; Chen, J.; Ostrikov, K. Emerging Energy and Environmental Applications of VerticallyOriented Graphenes. Chem. Soc. Rev. 2015, 44, 2108-2121.

(2) Sivadasan, A. K.; Parida, S.; Ghosh, S.; Pandian, R.; Dhara, S. Spectroscopically Forbidden Infra-Red Emission in Au-Vertical Graphene Hybrid Nanostructures. Nanotechnology 2017, 28, 465703.

(3) Vizireanu, S.; Dinescu, G.; Nistor, L. C.; Baibarac, M.; Ruxanda, G.; Stancu, M.; Ciuparu, D. Stability of Carbon Nanowalls Against Chemical Attack with Acid Solutions. Phys. E 2013, 47, 59-65.

(4) Li, L.; Dong, Y.; Guo, W.; Qian, F.; Xiong, F.; Fu, Y.; Du, Z.; Xu, C.; Sun, J. High-Responsivity Photodetectors Made of Graphene Nanowalls Grown on Si. Appl. Phys. Lett. 2019, 115, 081101.

(5) Davami, K.; Jiang, Y.; Cortes, J.; Lin, C.; Shaygan, M.; Turner, K. T.; Bargatin, I. Tuning the Mechanical Properties of Vertical Graphene Sheets Through Atomic Layer Deposition. Nanotechnology 2016, 27, 155701 .

(6) Qian, F.; Deng, J.; Xiong, F.; Dong, Y.; Hu, L.; Pan, G.; Wang, Q.; Xie, Y.; Sun, J.; Xu, C. Direct Growth of High Quality Graphene Nanowalls on Dielectric Surfaces by Plasma-Enhanced Chemical Vapor Deposition for Photo Detection. Opt. Mater. Express 2020, 10, 2909-2918.

(7) Zhou, Q.; Liu, X.; Zhang, E.; Luo, S.; Shen, J.; Wang, Y.; Wei, D. The Controlled Growth of Graphene Nanowalls on Si for Schottky Photodetector. AIP Adv. 2017, 7, 125317.

(8) Wang, J. J.; Zhu, M. Y.; Outlaw, R. A.; Zhao, X.; Manos, D. M.; Holloway, B. C.; Mammana, V. P. Free-Standing Subnanometer Graphite Sheets. Appl. Phys. Lett. 2004, 85, 1265-1267.

(9) Bo, Z.; Yu, K.; Lu, G.; Cui, S.; Mao, S.; Chen, J. Vertically oriented graphene sheets grown on metallic wires for greener corona discharges: lower power consumption and minimized ozone emission. Energy Environ. Sci. 2011, 4, 2525-2528.

(10) Vyas, A.; Cornaglia, F.; Rattanasawatesun, T.; Li, Q.; Haque, M.; Sun, J.; Kuzmenko, V.; Smith, A. D.; Lundgren, P.; Enoksson, P. Investigation of Palladium Current Collectors for Vertical GrapheneBased Microsupercapacitors. J. Phys.: Conf. Ser. 2019, 1319, 012007.

(11) Chen, J.; Bo, Z.; Lu, G. Vertically-Oriented Graphene-PECVD Synthesis and Applications; Springer: Switzerland, 2015; pp 19-34.

(12) Krivchenko, V. A.; Evlashin, S. A.; Mironovich, K. V.; Verbitskiy, N. I.; Nefedov, A.; Wöll, C.; Kozmenkova, A. Y.; Suetin, N. V.; Svyakhovskiy, S. E.; Vyalikh, D. V.; Rakhimov, A. T.; Egorov, A. V.; Yashina, L. V. Carbon nanowalls: the next step for physical manifestation of the black body coating. Sci. Rep. 2013, 3, 3328.

(13) Malesevic, A.; Kemps, R.; Vanhulsel, A.; Chowdhury, M. P.; Volodin, A.; Van Haesendonck, C. Field Emission from Vertically Aligned Few-Layer Graphene. J. Appl. Phys. 2008, 104, 084301.

(14) Ray, S. C.; Soin, N.; Makgato, T.; Chuang, C. H.; Pong, W. F.; Roy, S. S.; Ghosh, S. K.; Strydom, A. M.; McLaughlin, J. A. Graphene Supported Graphone/Graphane Bilayer Nanostructure Material for Spintronics. Sci. Rep. 2014, 4, 3862.

(15) Deng, J.-h.; Zheng, R.-t.; Zhao, Y.; Cheng, G.-a. Vapor-Solid Growth of Few-Layer Graphene Using Radio Frequency Sputtering Deposition and Its Application on Field Emission. ACS Nano 2012, 6, 3727-3733.

(16) Bo, Z.; Yang, Y.; Chen, J.; Yu, K.; Yan, J.; Cen, K. PlasmaEnhanced Chemical Vapor Deposition Synthesis of Vertically Oriented Graphene Nanosheets. Nanoscale 2013, 5, 5180-5204.

(17) Bisht, A.; Chockalingam, S.; Panwar, O. S.; Singh, B. P.; Kesarwani, A.; Chand, J. Synthesis of Vertical Graphene by Microwave Plasma Enhanced Chemical Vapor Deposition Technique. In Physics of Semiconductor Devices; Jain, V. K., Verma, A., Eds., 2014; pp 559-562. 
(18) Kim, Y.; Song, W.; Lee, S. Y.; Jeon, C.; Jung, W.; Kim, M.; Park, C.-Y. Low-Temperature Synthesis of Graphene on Nickel Foil by Microwave Plasma Chemical Vapor Deposition. Appl. Phys. Lett. 2011, 98, 263106.

(19) Zhu, M.; Wang, J.; Holloway, B. C.; Outlaw, R. A.; Zhao, X.; Hou, K.; Shutthanandan, V.; Manos, D. M. A Mechanism for Carbon Nanosheet Formation. Carbon 2007, 45, 2229-2234.

(20) Zhao, J.; Shaygan, M.; Eckert, J.; Meyyappan, M.; Rümmeli, M. H. A Growth Mechanism for Free-Standing Vertical Graphene. Nano Lett. 2014, 14, 3064-3071.

(21) Wu, Y.; Yang, B. Effects of Localized Electric Field on the Growth of Carbon Nanowalls. Nano Lett. 2002, 2, 355-359.

(22) Yu, K.; Wang, P.; Lu, G.; Chen, K.-H.; Bo, Z.; Chen, J. Patterning Vertically Oriented Graphene Sheets for Nanodevice Applications. J. Phys. Chem. Lett. 2011, 2, 537-542.

(23) Ma, X.; Qu, Q.; Zhao, Y.; Luo, Z.; Zhao, Y.; Ng, K. W.; Zhao, Y. Graphene Oxide Wrapped Gold Nanoparticles for Intracellular Raman Imaging and Drug Delivery. J. Mater. Chem. B 2013, 1, 64956500

(24) Pandit, S.; Cao, Z.; Mokkapati, V. R. S. S.; Celauro, E.; Yurgens, A.; Lovmar, M.; Westerlund, F.; Sun, J.; Mijakovic, I. Vertically Aligned Graphene Coating is Bactericidal and Prevents the Formation of Bacterial Biofilms. Adv. Mater. Interfaces 2018, 5, 1701331.

(25) Yu, K.; Lu, G.; Bo, Z.; Mao, S.; Chen, J. Carbon Nanotube with Chemically Bonded Graphene Leaves for Electronic and Optoelectronic Applications. J. Phys. Chem. Lett. 2011, 2, 1556-1562.

(26) Wang, X.; Liu, Y.; Zhu, D. A Perfect Carbon Nanotube with Two Closed Ends. J. Nanosci. Nanotechnol. 2002, 2, 33-35.

(27) Cai, M.; Outlaw, R. A.; Butler, S. M.; Miller, J. R. A High Density of Vertically-Oriented Graphenes for Use in Electric Double Layer Capacitors. Carbon 2012, 50, 5481-5488.

(28) Chen, H.; Wang, Z.; Zong, S.; Chen, P.; Zhu, D.; Wu, L.; Cui, Y. A Graphene Quantum Dot-Based FRET System for NuclearTargeted and Real-Time Monitoring of Drug Delivery. Nanoscale 2015, 7, 15477-15486.

(29) Frisch, M. J.; Trucks, G. W.; Schlegel, H. B.; Scuseria, G. E.; Robb, M. A.; Cheeseman, J. R.; Scalmani, G.; Barone, V.; Mennucci, B.; Petersson, G. A.; Nakatsuji, H.; Caricato, M.; Li, X.; Hratchian, H. P.; Izmaylov, A. F.; Bloino, J.; Zheng, G.; Sonnenberg, J. L.; Hada, M.; Ehara, M.; Toyota, K.; Fukuda, R.; Hasegawa, J.; Ishida, M.; Nakajima, T.; Honda, Y.; Kitao, O.; Nakai, H.; Vreven, T.; Montgomery, J. A., Jr.; Peralta, J. E.; Ogliaro, F.; Bearpark, M.; Heyd, J. J.; Brothers, E.; Kudin, K. N.; Staroverov, V. N.; Kobayashi, R.; Normand, J.; Raghavachari, K.; Rendell, A.; Burant, J. C.; Iyengar, S. S.; Tomasi, J.; Cossi, M.; Rega, N.; Millam, J. M.; Klene, M.; Knox, J. E.; Cross, J. B.; Bakken, V.; Adamo, C.; Jaramillo, J.; Gomperts, R.; Stratmann, R. E.; Yazyev, O.; Austin, A. J.; Cammi, R.; Pomelli, C.; Ochterski, J. W.; Martin, R. L.; Morokuma, K.; Zakrzewski, V. G.; Voth, G. A.; Salvador, P.; Dannenberg, J. J.; Dapprich, S.; Daniels, A. D.; Farkas, Ö.; Foresman, J. B.; Ortiz, J. V.; Cioslowski, J.; Fox, D. J. Gaussian 09; Gaussian, Inc.: Wallingford CT, 2009.

(30) Zhu, H.; Zhu, J.; Zhang, Z.; Zhao, R. Crossover from Linear Chains to a Honeycomb Network for the Nucleation of Hexagonal Boron Nitride Grown on the Ni(111) Surface. J. Phys. Chem. C 2021, $125,26542-26551$.

(31) Merenkov, I. S.; Kasatkin, I. A.; Kosinova, M. L. X-Ray Diffraction Study of Vertically Aligned Layers of h-BN, Obtained by PECVD from Borazine and Ammonia or Helium Mixtures. J. Struct. Chem. 2015, 56, 1173-1175.

(32) Bolhuis, M.; Hernandez-Rueda, J.; van Heijst, S. E.; Tinoco Rivas, M.; Kuipers, L.; Conesa-Boj, S. Vertically-Oriented $\mathrm{MoS}_{2}$ Nanosheets for Nonlinear Optical Devices. Nanoscale 2020, 12, 10491-10497.

(33) Sari, F. N. I.; Ting, J.-M. Direct Growth of $\mathrm{MoS}_{2}$ Nanowalls on Carbon Nanofibers for Use in Supercapacitor. Sci. Rep. 2017, 7, 5999.

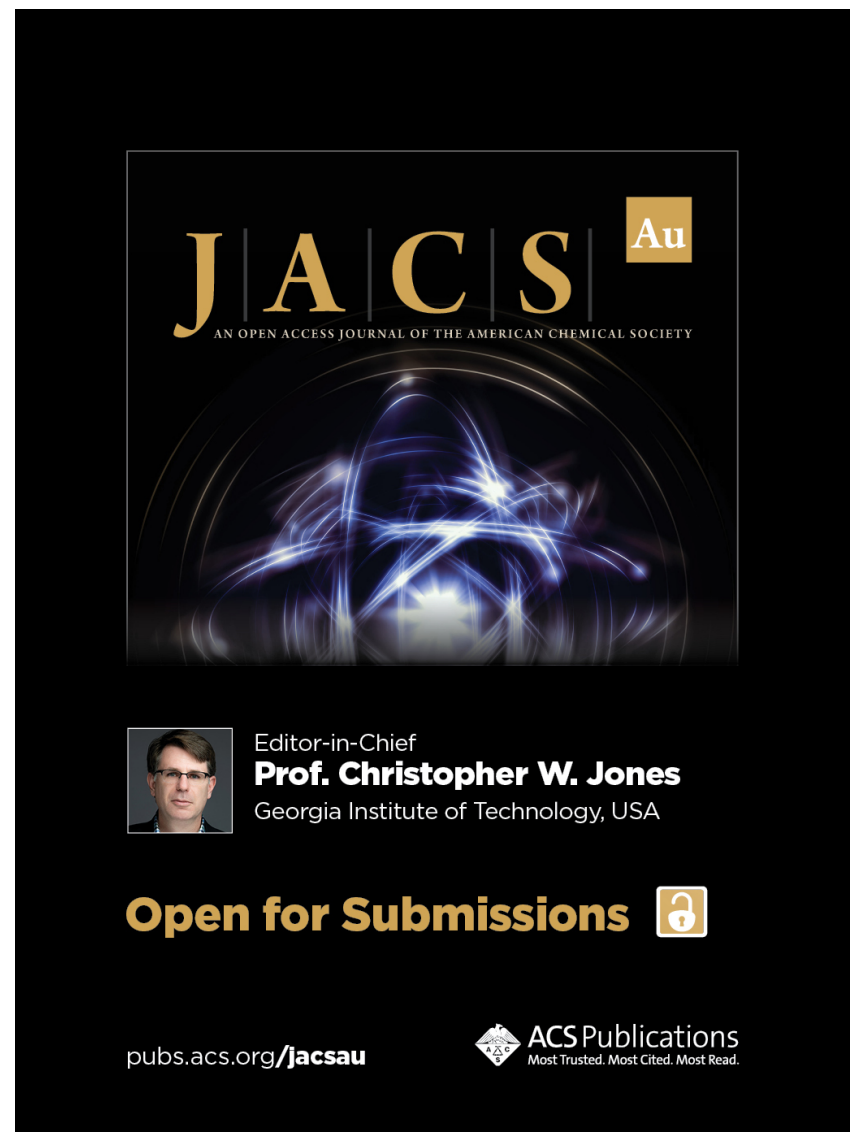

https://doi.org/10.1021/acsami.1c21640 ACS Appl. Mater. Interfaces 2022, 14, 7152-7160 